\title{
COMPUTER APPLICATIONS IN CONTROLLED THERMONUCLEAR RESEARCH
}

\author{
John Killeen
}

August 15, 1973

Prepared for U.S. Atomic Energy Commissionunder contract No. W-7405-Eng-48

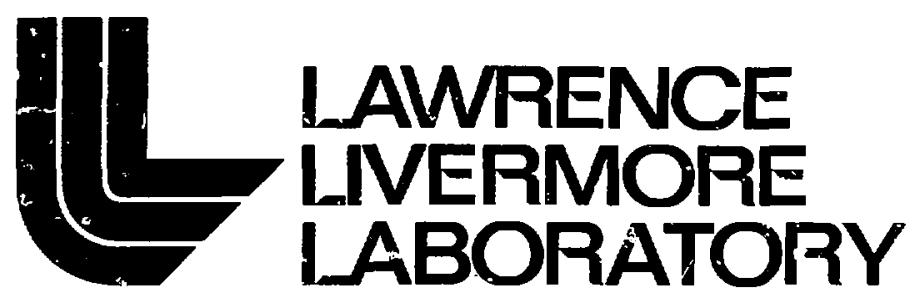

University of California/Livermore 
NTIXT.

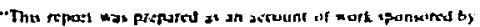

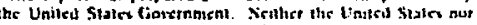

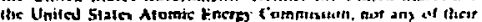

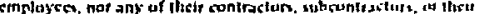

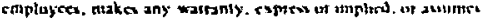

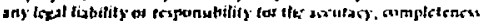

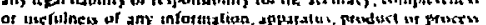

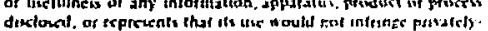
ongrd tithis."

Printed in the United States of America Available from

National Technical Information Service

U.S. Department of Commerce

5285 Port Royal Road

Springfield, Virginia 22151

Price: Printed Copy $\$$ : Microfiche $\$ 0.95$

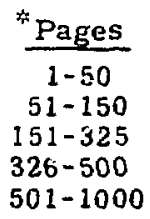

NTIS

Selling Price

$\$ 4.00$

$\$ 5.45$

$\$ 7.60$

$\$ 10.60$

$\$ 13.60$ 
TID-4500, UC-20, Controlled Thermonuclear Processes and Plasma Physics

\title{
노 \\ LAWFENCE UNERMOAE LABORATOFY

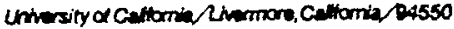

UCRL-51439

\section{COMPUTER APPLICATIONS IN CONTROLLED THERMONUCIEAR RESEARCH}

\author{
Joh: Killeen
}

MS. date: Auglist 15, 1973

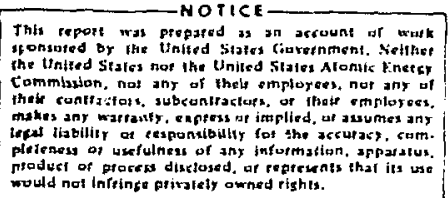

Summary of a talk given at the

1 th Student-Faculty Nuclear Engineering Conference

Argonne National Laboratory

Aug. 13-17, 1973 


\section{Contents}

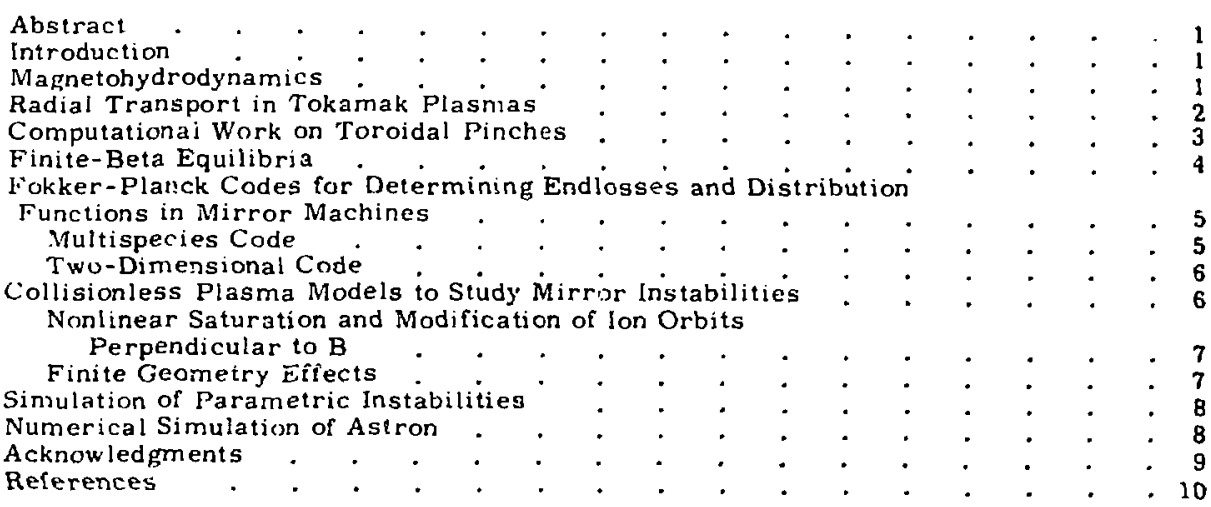




\title{
COMPUTER APPLICATIONS IN CONTROLLED THERMONUCLEAR RESEARCH.
}

\begin{abstract}
This report surveys those areas of plasma physics and cortrolled thermonuciear research in which computation has made significant contributions. Both

fluid and particle models are considered, and applications to specific confinement exferiments and plasma theory are discussed.
\end{abstract}

\section{Introduction}

Numerical calcula.ions have had an important role in controlled thermonuclear research since its beginning in the early $1950 ' \mathrm{~s}$, but the application of computers to plasma physics has advanced rapidly $i_{1}$ : the iast few years. Since 1967 there have been six conferences devoted to the Numerical Simulation of Plasmas, and during that same period the CTR computer usage has increased by nearly an order of magnitude. One reason for this is the increasing sophistication of the mathematical models of plasma behavior, and another is the increased speed and memory of the computers which made it reasonable to consider numerical simulation of plasmas.

A general reference for this subject is my Scottish Universitics Summer School lecture notes. Although these lectures were given in 1968 , the discussion of mathematical models of plasma confined by magnetic fields is appicable, and the numerical techniques, e. g. . difference methods for solving partial differential equations, are now widely used. In 1970. Volume 9 of the series of books titled "Methods in Computational Physics" was devoted to plasma physics.2 Most of the book is devoted to articles on the solution of the Vlasov or collisionless Boltzmann equation, and these include the manyparticle simulation techniques. There are two chapters on collisional plasma models - - one on the numerical solution of the Fokker-Planck equation for a plasma, and one on magnetohydrodynamic calculations. Another general reference is the "Proiseedings of the Fourth Conference on Numerical Simulation of

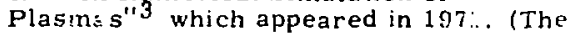
proceedings of the other five Confurences contain only abstracts.;

In this survey we shall consider the following areas in which computation has made significant contributions:

1. Magnetohydrodynamics

2. Radial Transport in Tokamak Plasnua:

3. Computational Work on Toroidal Pinches

4. Finite Beta Equilibria

5. Folker-Planck Codes for the Determination of Endlosses in Mirror Machines

6. Collicionless Plasma Models to Study Mirror Instabilities

7. The Simulation of Paranietric Instabilities

8. Numerical Simulation of Astron.

\section{Magnetohydrodynamics}

Many mathematical models are used to describe a plasma in a magnetic field, and these vary from the description of single-particle orbits to the kinetic theory of ionized gases. The fluid or magnetohydrodynamics (MHD) model is basic, and any experiment is first analyzed to determine if MHI equilibria and stability exist. Within the fluid theory various degrees of complexity are cousidered. The so-called ideal MHD is an infinite conductivity approximation. In some 
models the pressure is a scalar function, but in some problems it is necessary to consider it as a tensor with different values along and perpendicular to the magnetic field. The more realistic models include the transport coefficients, e. g., thermal conductivity and electrical resistivity, and these can also be scalars or tensors.

The article by Roberts and Potter ${ }^{4}$ gives a good review of the role of MHD computations and discusses methods for the solution of time-dependent problems. I have also $w$ ritten a review article ${ }^{5}$ for Information Processing 71 which considers both time-dependent and imeindependent problems.

The use of numerical calculations in the design and analy is of pinch experiments has been of cenisal importance. In most of these computations the equations of magnetohydrodynamics are used. The earliest problems ${ }^{6}$ used the infinite conductivity theory in analyzing the linear pinch. At Livermore, to analyze some experiments we coupled the pinch dynamica) equation with the external circuit equations. 7 The resulting set of differential equations were solved numerically.

As the stability theory of the pinch advanced and more complex experiments evolved such as the diffuse "stabi.ized" pinch, hard core pinch and theta pinch, more elaborate numıerical co:nputations have been made. Fortunately, at this same tine, the speed and capacity of computing machines have been increasing at a rapid rate.

Nlost of the above experiments hare been analyzed with computations on a tinie-dependent, one-dimensional, fully ionized hydromagnetic model. Electrical resistivity and thermal conductivit: of the plasma are included, and separate temperatures are assigned to the electrons and ions. The first programs with this model wero written by Haun and Roberts as a inint effort hetween AEIRF: Harwell and the Max-Pianck-Institut, Munich. In their work, Hhe equitions to be solved are given in Eulerian forn. Other programs whicl: hal been writtes. use the samc model s, but with equations in Lagransian form."

The resulting partial differential equa tions which describe the initia!-: alue problem are of both hypeibolic and parabolic type, and can be solved by difference methods which are described in Refs. 1, 4 and 5 .

To sive a better description of many of the experiments in CTR programs, a two-dimensional model is needed. We ronsider a cylindrical system with azimuthal symmetry. The variables are then functions of $r, z$ and $t$. Because of the success of the earlier calculations in describing certain experiments, we shall again consider a two-fluid model (unequal electron and ion temperatures) with thermal conduction. The velocity has components $v_{r}$ and $v_{z}$ and the magnetic field has components $\mathrm{B}_{r}$ and $\mathrm{B}_{z}$. This model is suitable for analyzing theta pinches, conical plasma guns, and the expansion of a laser-produced plasma in a magnetic field. A variety of difference schemes have been used and are described in Refs. 4 and 5 , An alternatingdirection inplicit code ${ }^{10}$ (ADI) has been developed and used to study theta pinch implosion and the expansion and trapping of a laser-produced plasma by an initially uniform magnetic field.

Another version of the two dimensional $\therefore$ z) MlHD equations use $v_{\phi}$ and $B_{\phi}$ inst sad of $v_{r}, v_{z}, B_{r}, B_{z}$ for describing the plasma focus experiments. Cor siderable success has been achieved in simulating inese experiments with both Eulerian ${ }^{4}$ and particle-in-cell $(P l C)^{11}$ schemes.

\section{Radial Transport in Tokamak Plasmas}

In the past few years, a considerable effort has been devotec to the numerical solution of radial transport equations for toroidal plasmas. This efiort proviues the only practical means of comparing thcory with experiment, and, moreover, is invaluable in optimizing the parameters for the design of future devices. This effort was recently reviewed by inchs 12 in a paper presented as the Garching Conference on Toroidal Confinement.
All of these computations are based on a description of the plasma by macroscopic variahles such as particle density $n$, electron and ion temperatures $T_{e}$ and $T_{i}$, and the poloidal and toroidal components of the magnetic field. T'ze cotes are one-dimensional and the cylindrical approximation is generally used, i.e., the magnetic surfaces arc assumed to be concentric circles and the cylindrical coordinate $r$ and the timo $t$ are the 
independent variables. There is currently some effort going into generalizing these codes to noncircular cross section.

In 1967 , we devcloped a code, 13 to solve the transport equations for $n(r, t)$ and $T(r, t)$. The dependence of the transport coefficients $c \mathrm{n}, \mathrm{T}$ and $\mathrm{B}$ u as allowed to be quite arbitrary so that we could study the sependence of the plasma profile on the form of the coefficients, and also look for thermal instabilities.

In 1968 the neoclassical transport theory 14 for Tokamak plasmas appeared which included the effects of trapped particles (hanana orbits) on the transport coefficients. Since 1969 a large number of numerical calculations have been reported at meetings based on models of increasing complexity. These are listed in the Düchs paper. 12
In 1971-72 the nrost complete sets of neoclassical transport equations were derived 15,16 [or both the banana and plateau regimes of collision frequency. There are now five codes - at Culham, 17 livermore, 17 Princeton, 18 Texas, 18 and Oak Riuge 19 - which include this most recent theory. The calculations have been checked against each other for a standard choice of initial and boundary conditions, and give equivalent results.

Considerable effort is now under way to generalize these codes to incluce the effects of neutrals, impurities, and empirical modeis of plasma transport 12,20 to give better agreement with experimental results from present day Tokamaks.

\section{Computational Work on Toroidal Pinches}

The objective of reversed field pinch experiments is to set up an MHD stable field configuration using field programming. Confinement is then limited primarily because field diffusion causes the configuraticn to decay until the stability properties are lost. In the analysis of both the setting up and decay phases, computational physics is playing an important role.

At the Garching meeting, Newton 21 described calculations which sirnulated the field programming (compression) phase up to the time that the crowtar is apnlied. The code that is used for this purpose is a generalization 22 of the original Hain-Roberts ${ }^{8}$ time-dependent, one-dimensional MHD code. The generalization is to a partially ionized gas, so it is a three-fluid model with classical transport coefficients except ior anomalous resistivity. I he code includes circuits for azimuthal and axial current with air core transformer coupling. Ionization and radiction are also included. Newtin stated that the inclusion of the full circuit and neutral atoms is necessary for a self-consistent calculation. Good agreement ir:as obtained between measured and compated field and density distributions.

The MHD stability of reversed field pinch configurations has been studied theoretirally by Robinson 23 using the hydromagnetic energy principle. In a paper at the Garching meeting, Grossman 24 described numerical calcuiations of MHD instability growth rates ior re- versed field profiles of the proposed Padua experiment which is under con. struction. The profiles considered correspond to various stages of the setting up (implosion) phase of the reversed field pinch. The profiles were obtained irom a one-dimensional, time-dependent code of the type described in the previous paragraph. The instability growth rates were obtained by solving the linearized ideal MHD equations including compressibility. The effect of compressibilıty is to raise the growth rates of urstable modes.

In the analysis of the decay phase af reversed field pinches, several codes are in use at Culhan.. The results of these computations cari be compared with the existing $\mathrm{H} \beta \mathrm{TX}$ and can also aid in th a design of future toroidal pinch experiments. 25 To study the ef iects of coupled field, particle and thermal diffusion, a r.ew code originated by Killeen 26 has been developed at Culham and Livermore. It solves for $n(r, t)$, the number density of ions and electrons, $T_{e}(r, t)$ and $T_{i}(r, t)$, the electron and ion temperatures, and $B_{\theta}(r, t)$ and $B_{z}(r, t)$, the poloidal and toroidal compunents of the magnetic field. The classical transport coefficients have been used for the toroidal pinch calcuiations, but the code also includes the reoclassical (banana-plateau) coefficients. Results oi this code, showing the evolution of an initially stable reversed field pinch, were presented by Robinson 27 at the Garching meeting. These results also 
included a discussion of the thermal instability which shows up in some of the calcilations.

Three codes are in use at Culham for studying the stability of the evolving field configurations of the previous paragraph. One colle called NEWCOMB ${ }^{28}$ uses the idea. 'THD energy principle of the diffuse pinch 29 to determine marginal stability as a function of $m, k$ and wall radius, $\mathbf{R}_{w}$. Another code solves the linearized ideal MHD cquations for the growth rates of unstable modes. The third code in use is RIPFI.E. IV 30 which solves for the resistive tearing mode in cylindrical geometry for an arbitrary $B_{z}(r)$ and $B_{\theta}(r)$. Results of these codes for evolving reversed field profiles uere presented by Robins on 27 at the Garching meeting, and also at the Innsbruck meeting. 31

A nev: code 32 which solves for the resistive tearing mode in cylindrical geometry, but also including compressibility, therma! conductuvity, and viscosity, has been developed at Livermore. Preliminary results for reversed field configurations were presented at the Sherwood Theory Meeting in Austin, Texas, Marct, 197 ?

All of the codes cescribed above (woth diffusion and stability) are applicable to Tokamaks ${ }^{33}$ and other toroidai pinch plasmas.

\section{Finite-Beta Equilibria}

Experimental devices incorporating the idea of axial sym:netry in a torus appear to be capable of piasma confinement for times which are of great interest. One reason for this is the assurance of equilibria in such devices, as predictęd by MHD and guiding-center theories. 34 The choice of distribution function determines the type of equilibrium. With the guiding-center equations, one can choose the distribution function $f$ arbitrarily as a function of flux function $\psi$, magnetic moment $\mu_{r}$ and energy $\epsilon$. The components the pressure terisor $p_{\perp}$ and $p_{\|}$are then determined as moments of $f$, and can be written as functions of $\psi$ and magnitude of magnetic field $B$. The hydromagnetic equations then yield a nonlinear, secondorder partial differential equation for $\psi(r, z)$. We developed 35 the technique for solving such free boundary equilibria problems. A detailed description of this finite-difference method for solving the $\psi$ equation is given in Refs. 1 and 5.

Two-dimensional models for plasma equilibria have been used to compute the equilibrium fields for various containmert schemes including Astron, 36 Levitron, 37 Tokamak, and the stuffed cisp. 35 Several containment schemes. including the stabilized mirror devices. ùo not possess an ignorable coordinate, thus allowing a reduction of the equilibriun equations to two dimensions. A code 38 has been developed to ha dle the general case for open containme 1 . This is a three-dimerisional code that solves for plasma equilibria in open-field geometries which allows analysis of most minimum-B mirror systerms. Open confinement, with $p=p(B)$ only, requires the hydromagnetic equitibrium equation to reduce to one elliptic, scalar partial differential equation involving a tensor pressure. The finite difference form of the equation is solved by an implicit iterati re algorithm similar to the ADI (alternating direction implicit) method. Cylindrical grid coordinates are employed, and the mesh spacing is variable to allow representation of far boundary conditions. It is found that, when the threshold for hydromagnetic instability is passed, the hydromagnetic equilibrium equation becomes hyperbolic which leads to an ill-posed problem as predicted by Grad. 34 Equilibria are found for an increasing sequence of pressures until the instability threshold is reached. 'The present code utilized planes of inversion symmetry common to many minimum-B devices to aliow a reduction in the domain to be studied; I ife bar devices, Basebali II, $2 X-I I$ are representative. 


\section{Foklser-Planck Codes for Determining Endlosses and Distribution Functions in Mirror Machines}

\begin{abstract}
The proposition that a stable mirror plasma will yield net thermonuclear power hinges critically on the rate at which particles are lost out the ends of the device. In the absence of turbulence and at number densities and energies typical of mirror machines, the endloss $\epsilon$ are due primarily to the scattering of charged particles into the loss cones in velocity space by weak, classical Coulomb collisions. The kinetic equation describing this process is the Boltzmann equation with the Fokker-
\end{abstract} Planck collision term. 39

We are confronted, in principle, with one such nonlinear, partial, integrodifferential equation for each charged species in the plasma, and there are seven independent variables (three spatial coordinates, three velocities, and time). Such an equation, ever for a single species, exceeds the capability of any extant computer. Several sin:plifying assumptions are therefore required to treat the problem at 3.1 . However, the parallel improvement of computers and FokkerPlanck codes over the past 15 years has led to the progressive elimination of the most questionable approximations, and has made it possible to specify error brackets for some of the others. At (LLL), in particular, the strong experimental mirror programi and the unrivaled computing facilities have encouraged a major effort on the refinement of Fokins Planck codes. The two complementary codes described ir! the following paragraphs are, to our knowledge, the most versatile and powerful Fokker-Planck programs in operation today.

\section{MULTISPECIES COR ¿}

This code 40 can handle an arbitrary number of species of charged particles, and electrons are treated on equal footing with ions. The code has seen extensive use in determining endlosses and distribution functions under a wide range of hypothetical reactor conditions for both $\mathrm{D}-\mathrm{T}$ and $\mathrm{D}-{ }^{3} \mathrm{He}$ systems, including reaction products. 40 (Runs have been carried out with as many as five species -- D, e, ${ }^{3} \mathrm{He},{ }^{4} \mathrm{He}$, and $\mathrm{H}$-- mear.ing the simultaneous solution of five coupled FokkerPlanck equations.)

The principal approxinations in the multispecies code are as follows:
- The distribution functions are azimuthally and radially invariant in space and azimuthally invariant in velocity. This implies that the radius of the machine is much greater than a Larmor radius, and that the dominant loss process is along the field lines.

- The distribution functions are independent of the spatial coordinate along the field lines, as well. This follows if the mean free path of the particles greatly exceeds the length of the machine so that particles scattered into the loss cone in velority space are ejected immediately, and if the spatial variation of the confining magnetic field and ambipolar potential occurs only near the ends of the device. In a calculation of this kind which represents conditions in the uniform central region of a long mirror machine, the existence of the magnetic mirrors and ambipolar potential enters only through the boundary coriditions in velocity space. This is often called the "magnetic square well" approximation.

The numerical techniques by which the equations are solved have been described in detail elsewhere. 40 Briefly, the code employs an implicit finite-difference scheme, in which coefficients involving moments of the distribution functions are recomputed at each time step. The velocity mesh varies continuously to provide ample resolution over ion and electron distributions, and, in calculations seeking the equilibrium state corresponding to a given set of ion sources, the time step increases as the computation proceeds. An ambipolar potential is determined at each. time step; it serves to equalize loss rates of ions and electrons and is computed by requiring charge neutrality (to within a specified tolerance) in the bulk of the plasma.

The ILL multispecies code incorporates, in addition to the capability of hardling any number of species, several. other improvements over previous. separated Fokker-Planck treatments. The difference iorm of the terms containing velocity derivatives is explicitly particle-conserving, and both energy and particle conservation for each species are 
monitored in every mu. The errors are usually less than $1 \%$ and always less than $5 \%$. These results are useful not merely as diagnostics; they also provide insight into physical processes and may suggest routes to the optimization of reactor parameters. Specifically, matrices are printed out showing energy transfer rates among all species and the distribution of scattering losses of each species according to the species of the scatterers. Monitoring energy transfer is th: $s$ way has already led to the correction of an error (concerning the treatment of the Coulomb logarithm) evidently made in all previous multispecies calculations.

Another improvement over previous multispecies codes is in the computation of the fusion reaction-rate parameter, $\bar{\sigma} v$. A rigorous computation would require integration cver the full velocity space of both reacting ion species. Previous work with multispecies codes settled for approximating the first five integrations and performing only the last, over $v$ for one distribution numerically. The LLL multispecies code employs a triple integration incorporating the distributions of both reactants (variables kept are the magnitudes of the two velocities and the magnitude of the relative velocity?.

To date, the multispecies code has been used to determine equilibrium conditions, distribution functions and values of $Q(=$ fusion power/injection power) for $D-T$ systems with injection energies from 100 to $800 \mathrm{keV}$, and for $\mathrm{D}-{ }^{3} \mathrm{He}$ systems with injection energies from 300 to 1500 $\mathrm{keV}$ and a variety of $3_{\mathrm{He}}$ fractions. Sensitivity to mirror ratio. fusion crosssection data, and the inclusion of reaction products have been tested. 40 The code is also equipped to treat buildup and decay problems, charge exchange, and multiple ion beam injection at different el.ergies. Gutilt quantities displayed graphically include the evolution in time of densities, mean energies, and the distribution functions themselves, as well as the energy spectrum of the particles actually escaping through the mirrors. The latter information is essential to the detailed design of direct conversion schemes.

\section{TWO-DIMENSIONAL CODE}

A two-dimensional Fokker-Planck code $^{41}$ is in routine operation at LLL. This code solves the unseparated FokkerPlanck equation in $v$ and $\theta$ for a single ion species, under the assumption that the electrons can be represented by a Maxwellian distribution function with loss cone removed. The assumption of azimuthal and radial invariance in space and azimuthal invariance in velocity is rotained.

The ion equation is solved using the alternating-direction implicit (ADI) finite difference scheme $e^{41}$ ca a variable mesh in $v$ and $\theta$, and the coefficients containing moments over both ion and electron distribution functions are recomputed at each ion time step. The number density and mean energy characterizing the Maxwellian distribution of electrons are computed on a faster time scale by solving a pair of ordinary differential equations that include the effects of ion-electron interactions and electron endlosses and injection conditions. The ambipolar potential is determined by iteration on the electron equations in such a way as to equalize the positive and negative charge densities, as in the multispecies code.

The two-dimensional code can also treat realistic spatial dependence of the magnitude of the confining magnetic field ( $z$-dependence), as well as spatially distributed sources. In the $z$-dependent version of the code, the spatial variation of the ambipolar potential is a computed quantity. 41

To date, the two-dimensional code has been used extensively in buildup calculations corresponding to the Alice experiment, in decay calculations corresponding to the $2 \mathrm{X}$ experiment, and finally, in steady-state calculations under proposed reactor conditions comparable to those investigated with the multispecies code. Some of these results have been described elsewhere. 41 Results as yet unpublished include the effects of charge exchange and of timevarying magnetic fields.

\section{Collisionless Plasma Models to Study Mirror Instabilities}

The Vlasov equation is one of the principal mathematical models used in plasma physics. Techniques for the numerical solution of a Vlasov system in both one and two dimensions are de$\mathrm{sc}$.ibed in Refs. 1 and 2. Most of the 
codes use n J.agranguian schems, i. e., the plasma is represented by a large number of "particles" which carry" charge and mass, and the self.consistent electric and magnetic fields are computed from the calculated motion of the particles. Some applications of these codes to the study of plasma instahilities in mirror machines are discussed in the following.

\section{NONIINEAR SATURATION AND \\ MODIFICATION OF ION ORBITS \\ PER PENISIC:LITAR TO $B$}

One group of codes primarily described the ion dynamics perpendicular to $B$ for a variety of flute modes, all expected or actually observed in actual mirror devices: Dory-Guest-Harris loss cone, double hump (due to impurities or the presence of a cold target plasma), and drift cone. The flute modes are expected to persist in any mirror device, regardless of its length. The drift cone mode, in particular, remains as a major theoretical stumbling block to stable mirror confinement.

Most of the results obtained contributed to basiz plasma physics, specifically the understanding of nonlinear saturation mechanisms. In particular, the codes confirmed in detail the theoretical caiculations for the saturation of certain modes. In addition, the codes determined the saturation mechanisms for the other flute modes not amenable to analys is 42,43

These codes also confirmed the presence of and described the saturation of 3 -wave and 2 -wave particle nonlinear explosive instabilities. Of significance here was the finding that these modes are extremely sensitive to resonance conditions, and hence are unlikely to be a threat to confinement. 44

One major code result aided in the interpretation of the rf spectra obtained from $2 X$, which were dominated by signals near the ion cyclotron harmonics. The code results showed that the nonlinear saturation always involved a defirite change away from the linear regime real frequency, and that frequently there occurs a spectrum dominated by signals near the harmonics, despite the presence of modes whose linear real frequencies were nonharmonic. Thus, whatever the linitations of the model and whatever the precise saturation nuechanism in the actual experiment, the code results show that the presence or absence of spectra specifically tied to linear theory is likely not to be a useful diagnostic in proving the existence or absence of sperific modes. 45

In sum, these codes provided the first detailed look at the microturbulence resulting from high-frequency mirror instabilities, and specifically:

1. Produced direct ties to analytical calculations.

2. Produced significant results unobta inable by other mears on nonlineat saturations mechanisms, and predicled that the nonlinear saturation levels obtained by flute modes saturated by ion orbit changes would likely be disastrous for confinement.

3. Predicied the relative unimportance of the "explosive" nonlinear instabilities, despite their presence under highly idealized conditions.

4. Had direct meaning for interpretation of $2 X$ rf spectra.

\section{FINITE GEOMETRY EFFECTS}

Another group of codes examined finite geometry effects on the flute modes. A 2-1/2 dimensional particle simulation code examined the nonlinear saturation of the modified negative mass in finite length models. 46 This mode requires rather singular velocity distributions. and can easily be cured in practice.

A linearized code examined finite geometry effects on flute modes in idealized slab geometry, 47 with the following results:

1. Confirmed the idea that short lengths do not seriously affect flute modes;

2. showed that flute mode potentials hold up to points where the densify is a very small fraction of its midplane value; and

3. produced some information on the behavior of the drift cone mode in a syster, with a finite cross section.

A one-dimens th coll coxamined the nonlinear behavic of electrons responding along the $B$ lines to an unstable flute mode. $48^{\circ}$ As expected, gross modification of the mode occurs when e $\phi_{\text {wave }}=\mathrm{T}_{\mathrm{e}}$; more work is required to determine whether this is a true saturation mecha nism. The basic effect is expected :c opsrate in any unstable flute mode, and will have to be accounted for in future models.

A single-particle code followed particle orbits in mirror magnetic fields with arbitrarily assumed fluctuating 
electric fields. The main result of this code was that particle confinement can persist for long times (many bounce times), despite the presence of flute-like electric fields that produce changes in the particle's magnetic moment. These chariges momentarily place the velocity vector in the loss cone. Also, strongly nonflute fields produce rapjel loss of particles. This result provides one possible explanation for the long life of $2 x$ despite conditions that are strongly unstable to the drift cone mods: that is, if the mode is present at some undetermined nonlinar level but remains flute-like, thr particles will still be contanned for long times. The time seale for confinement depends in detall on the strength and frequeney spectrum of the fluctuating elecetric ric.lds 49

\section{Simulation of Parametric Instabilities}

Parametric instabilities involve a periodic modulation of the plasma by an electromagnetic was $\&$ or modulated electron beam. They are of interest primarily as heating mechanisms. The externat energy source ("pump") drives two waves in the plasma whose frequencies and wave vectors add up to those of the pump. In the pseudn-quantum mechanical description, a pump quantum decays into two lower-frequency wave quanta. The matching of wave vectors and frequencies corresponds to the conservation of the momentum and energy of the decaying pump quantum. There also are analogies to an important class of electronic amplificrs.

Early simulations were of the ionacoustic decay anci oscillating two-stream instabilities in which the pump is a (nearly) uniforn oscillating external electric field. $50-55$ This represents the electromagnetic wave when it has traveled into the "critical density" surface where the laser frequency equals the local plasma frequency. Plasma oscillations grow and heat electrons by trapping or quasilimear diffusion, depending on the amplitudes and spectrum of the plasma waves. This was the first heating process, other than collisions (inverse bremsstrahlung), considered in laserinduced fusion, and is sometimes called "anomalous" heating. Some ion heating also can occur. Simulations are used to estimate he ating rates and the resultant electron enelgy spectrum.
Of particulat interest in ("Th applications are parametric processes in which the pump frequency is much lower, around ion wave frequencies. Examples irclude driving a lower-hybrid oscilla. $n^{56}$ and the mndified iwo-stream. 57 Arother example is the deciay of a whistler. 58 Such processes offer the possibility of heating jons in magnetically contined plasmas.

Parametric instabilities also oceur in underdense plasmas. One wave may be an electron oscillation or an ion-acoustic wave, and the other is an electromagnetic wave. These cases are commonly called srimulated Raman and Brillnuin scattering, in arsilogy to processises in cither media. Tney are of great interest to laser fusion because they may cause a large part of the laser energy to be lost by scattering, and vecause of the clectron heating by Raman's electron decay wave. 59,60 Decay into two electromagnetic wayes is not possible in an unmagnetized plasma because irequencies and wave vecturs cannot simultaneously be matched. Another process is the decay into two electron plasma osicillations, which provides an alternative heating mechanism occurring at one-quarter critical density.61,62 In the presence of self focusing or filamentation of the laser light additional decay possibilities arise. Simulations are used to study rates and efficiencies of heating, effects of density gradients, laser frequency bandwidth, etc.

\section{Numerical Simulation of Astron}

A time-dependent numerical model of the Astron, with which injection and trapping can be studied in detail, has been developed. The effects due to the resistors and neutralization have been in- cluded. The model is axially symmetrie. The E-layer clectrons are simulated by many thousands of finite-size superparticles, which move in the $r-z$ domain and have velocity components $v_{p}, v_{\theta}$, and $v_{z}$. 


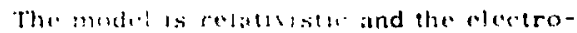
magnetal felds are whotued by solving four wase equatuons - - three for the recter potential and one for the sealat potential. The E-layer current and the current thduced in the resistor wires are included in the above feeld rqualions. The romputed self-fields are added to the estremal field to give the ficld configutation as a fum.tion of time. Results of multiple guise injerion have becn published. 64

The code resulis have heen continually and intimately involved in interpretations of and deopsirns about the Astron experiments, 65

- In the lery rirst series of runs on the Superlayer code, it phenomenon of saturation of the field reversal, $\zeta$ was discoverted. This phenomenon was due to an axial lengthering during pulse sta:king under charge neutralized conditions. This result directly stimulat ed other theoretical work, and the two logether played a large part in the decision to turn the Astron experiment to stacking in vacnum, which was continded fot more than a rear. This lenghening phenomenon was discovered firs: by the code, and only lator was obscred in the experiment.

- The code reprodiced several experinental results of trapping cfficiency in vacum; in particular, the trapping efficiency in a particular magnetic well configuration was sensitive to parameter changes (resistor location, injection cur rent) in the same way as he experiment. thus providinf the assurance of a simple explanation unrelated to difficulties peculiar to the cxperinent.

- The code rosults show that stacking in racuum ultimately causes difficulty at sufficienily high $\zeta$, in the range 0.5 to 1.0 . Jue to increased radial expansion. This has yet to be confirmed by the experument since they are still at low s. but this result is one factor that must be considered in any plans to stack to reversal in vacuum. This result is modified under conditions of partial neulralizaturn, such as exists experimentally even under the best vacuum.

- Reversal is obtained starting with vacuum stacking of 1000-A pulses up to the point of incipient radial loss followed by adiabatic charge neutralization (which compresses the layer and nearly tinubles the field reversalf, but this route may well not be successful when the idealized code conditions are relaxed.

- The code shows that sufficiently large rurrent lonly 4 times that previously available in the experiment and possibly only 2 times that available with the spli: pulse) can produce field reversal in one pulse (in charge neutralized environmeni and in a short system). This route does not appear sensitive to relaxation of idealized code conditions.

- The efficiency of single-pulse trapping in the code for a wide variety of conditions is nearly perfect. This fact explicitly tells us that the experimental poor trapping efficiency is due to nondeal conditions such as instabilitjes due to $r, \theta$ notion (see below) or to combined effects of poor beam quality and asymmetries in the magnetic fields. This latter effect has recently been confirmed in the experiment where the poor trapping was found in part to be caused by fross field asymmetries at the injector

The results obtained from an $(r, \theta)$ code show that for a mojerate value of $\zeta \geq 0.1$, in an unneutralized layer. the negative mass instability will result in large radia! loss, and thus prevent buildup in vacum in reversal levels. 66 Other versions of this code are capable of examining nonideal effects such as $z$-variation and conplex equilibrium orbits. 66

\section{Acknowledgments}

I wish to thank Jack Byers, John Holdren, Bruce I angdon and Brendan Mlsima, a for holp in preparieg this survey. 


\section{References}

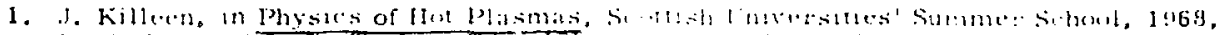

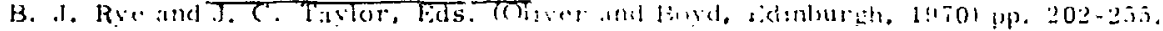

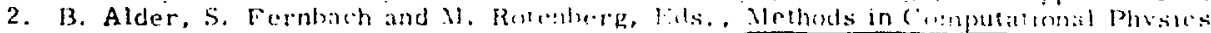
Academic J'ress, New lork, 1970 serl.!?

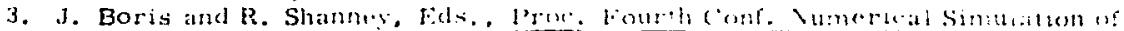

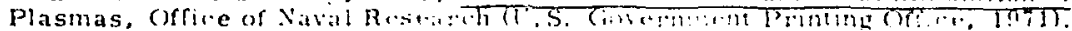

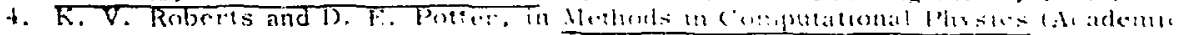

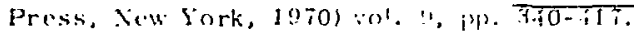

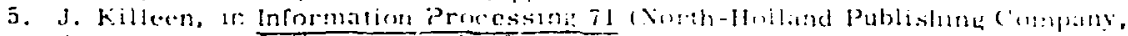
Ansterdam, 1972) pl. 11!1-1205.

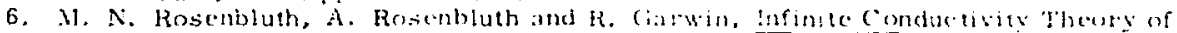

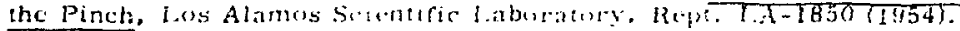

7. T. Filleen and B. A. I.ipmann, I. Appl. phys. $31,1542(1560)$.

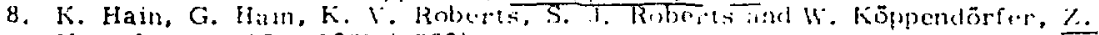
Naturforsch. 15a, 1030 (1960).

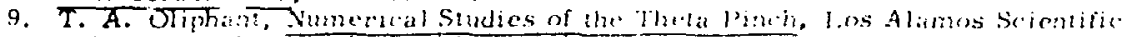

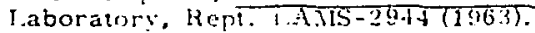

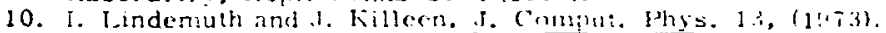

11. R. Morse, in Methods in Computational Physies TAratcmic Pross, New ropk, 1970) rol. 9 .

12. D. Duchs, H. P. Furth and P. H. Rutherturd, in Proc. Third Intern. Symp.

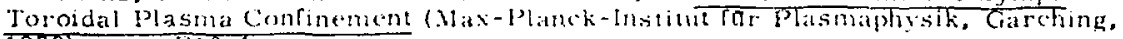
1973) paper $1310-1$.

13. J. Killeen, H. P. Furth, K. 1). Narx and M. A. Rosenbluth, Bull. Am. Plyss. So:. 13, 26? (1068).

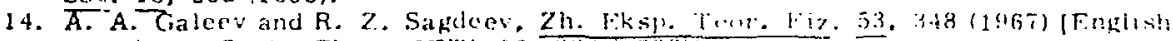

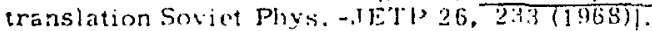

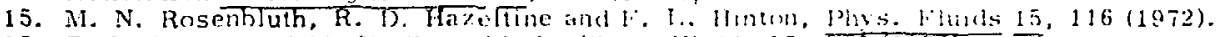

16. F. [. Finton and .1. N. Rosenbluth, l'hys. Fluids 16, T36 (1973).

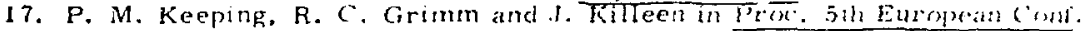

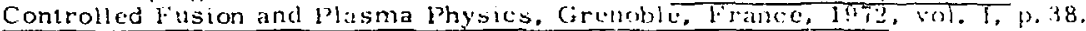

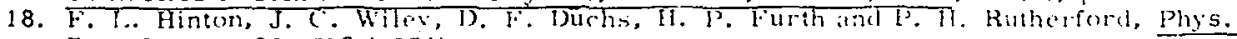
Rev. l.etters 29,698 (1)

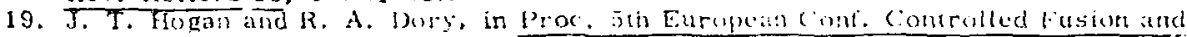
Plasma Physics, Cirenoble, France, 1972, col. 1. p. 40.

20. G. C. Kelley ". al. in Pror. Third lntern. Symp. Torndial Plasma Confinement

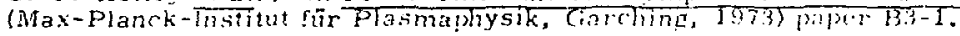

21. J. E. Crow et al. , ibid. , paper As.

22. K. V. Roberts, Platima Phys. 5, $365(1063)$.

23. D. C. Robilison, 1गasmit Phys. 13,439 (1071).

24. S. Ortolani and W. Grossman, in Pro. Thurl Intern. Symp. Toroidial plasma

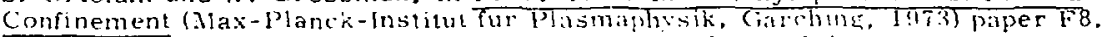

25. H. A. B. Bodin, T. E. lames, A. A. Newton and D. (C. Rehunson, lbid, paper G3.

26. J. Killeon, The Nume riall sulutum of Rurlal Transpor Fquations for Toroidal

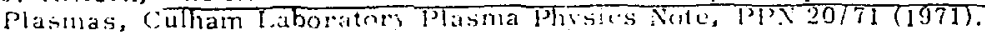

2i. T. F. Crow and D. C. Rnbinsom, in Proc. Thard Intern. Symp. Toroidal Plasma Confinenent (Max-Planck-Institut für Pasilidphysik, (iarching, 1973) paper A4.

28. D. M. Copley and K. J. Whiteman, Plasmit Phys. 4. 10341962$)$.

29. W. A. Newcomb. Ann, Phys. 10, 232 1060 .

30. J. Killeen, "Resistive Instability Calculations," in Jhysics of Hot Plasmas. 13. J. Rye and J. C. Taylor, Eds. (C)liver and Boyd, Edithumb, 1970) pp. 212-225.

31. 1. E. Crow and 1 , C Robinson, in Proc. Interm. (angress on Waves and Instabilitues in Plasmas, Innsbruck, Austrit, April 2-6, I!73, Paper ('6.

32. J. Dibiase and I. Killeen, Sherwond Theort blethin, Austin, rexas, March $12-13,1073$.

33. 3. E. Crow, 1. Killeren and 1). (C, Robinsm, paper submbted to 6 th Furoprean Comf.

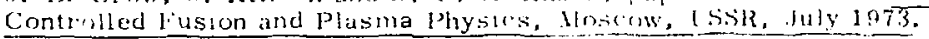


34. H. Civid, l'tys. Fluids 10,137 (1967).

35. A. Killew indk. T. Whiteman, Phys. Fluids 9, 1846 (1966).

36. 11. 1. Anderson. J. Killecn, and M. E. Rensiñk, Phys. Fluids 15, 351 (1972).

37. S. Fisher and I. Killecn. Phys. Fluids 14, 1240 (5971).

38. D. V. Anderson and .1. Ki Teen, J.C.omput. Thys. 10, 133 (1972).

39. .1. N. Rosenisluth, W. M. MacDonald andD. L.. Judd. Phys. Rev. 107. 1 (1957).

40. A. H. Futcl, Jr., I. P. Holdren, I. Killecn and A. A. Mirin, Plasma Phys. $1+, 211(1 ! 17 \geq)$.

41. J. Killeen ind K. D. Marx, "The Solution of the Fokker-Planck Equation for a Mirror-Confined Plasma," in Methods in Computational Physics (Academic Press, Virw lork, 1070) vol. 9, pp. $421-489$.

42. J. A. Byers and $M$, Grewal, Phys. Fluids 13, 1819 (1970).

43. C. K. Bircisall and D. Fuss, Ion Cyclotron Wave Growth and Saturation in a Uniform Plasma Simulated by a Two-Dimensional Computer Model, L.awrence livermore l.abriatory, Rrpt. UCR!-73983 (1972), submilled for publication in Phys. Fluids.

44. J. A. Byers, M. E. Rensink, J. I.. Smith and G. M. Walters. Phys. Fluids 14, $826(1.971)$.

45. S. E. Bòdrer and J. A. Byers, Phys, Rev. Letters 23, 967 (1969).

46. D. Fuss and B. McNamara, "Direct Sinulation of Plasma Instabilities in Mirror Machires," in CTR Annual Report July 1970-June 1971. [.awrence Livermore I.aboratory, Rept. TCRL-50002-71 (1971) p. 1-2.

47. J. A. Byers, "Mirror Flute Mode Behavior in Finite Geometry," ibid. . p. 1-9.

48. H. C. Burk and R. C. Harding, "Simulation of Loss-Cone Modes in Inhomogeneous Geonsetry, " in CTR Annual Report July 1971 -June 1972, Lawrence i.ivermore L.aboratory, Rept. UCRL-50002-72, p. 15.

49. R. E. Aamodt and J. A. Byers. Phys. Rev. Letters 29, 1305 (1972).

50. W. Kruer, P. Kaw, J. Dawson and C. Oherman, Phys. Rev. L.atters 24, 987 $(1070)$.

51. W. Kruer and J. Dawson, Phys. Fluids 15, 466 (1972).

52. J. S. DeGroot and J. I. Katz, Phys. Fluids 16, 40) (1973).

53. 5. E. Bodner, G. F. Chapline and J. DeGroot, Plasma Phys. 15. 21 (1973).

54. B. Godfrey, $C$. Rhoades, and $k$. Taggart, "Conputer Simulation of the Saturation of the Parametric instability in the Weak Turbulence Region," AFWLTR-72-22B (1973), to be published in Phys. Fluids.

55. J. J. Thomson, R. J. Faehl and W. I.. Kruer, "Mode Coupling Saturation of the Parametric Instability and Electron Heating," to be published in Phys. Rev. Letiers.

56. J. M. Kindel. H. Okuda and I. M. Dawson, Phys. Rev. I.etters 29, 995 (1972).

57. L. Chen and C. K. Birdsall, "Heating of Magnetized Plasmas by a LargeAmplitude, Low-Frequency Electric Field, "to be published in Phys. Fluids.

58. D. W. Forslund, J. M. Kindel and E. I. Lindman, Phys. Rev. Letters 29, $249(1972)$.

59. D. W. Forslund, J. M. Kindel, and E. L. Lindman, Phys. Rev. Letters 30 $739(1973)$.

60. W. I.. Kruer, K. G. Estabrook, and K. H. Sinz, instability-Generated Laser Reflection in Plasmas, Lawrence Livermore Laboratory, Rept. UCRL-74676, to be published.

61. W. I.. Kruer and J. M. Dawson, Phys. Fluids 14, 1003 (1971).

62. A. B. Langdon, B. F. Lasinski, and W. L. Kruer, Plasma Heating at OneQuarter Critical Density, to be published.

63. A. B. Laıgdon and B. F. I a sinski, "Filanientation and Subsequent Decay of I aser l,ignt in Plasmas," to be published.

64. M. Brettschneider, J. Killeen and A. A. Mirin, J. Comput. Phys. 11, 360 (1973).

65. J. A. Byers, J. P. Holdren, J. Killeen, A. B. Iangdon, A. A. Mirin, M. E. Rensink and C. G. Tull, "Computer Simulation of Pulse Trapping and Pulse Stacking of Reidivistic Electron Layers in Astron," to be submitted for publication in Phys. Fluids.

66. M. Cirewal and J. A. Byers, Plasma Phys. 11, 727 (1969). 